EPJ Web of Conferences 59, 13001 (2013)

DOI: $10.1051 /$ epjconf/20135913001

(C) Owned by the authors, published by EDP Sciences, 2013

\title{
VISAR diagnostic at LIL facility
}

\author{
S. Darbon ${ }^{1, a}$, A. Duval ${ }^{1}$, I. Masclet-Gobin ${ }^{1}$, B. Marchet ${ }^{1}$, S. Brygoo ${ }^{1}$, \\ C. Courtois ${ }^{1}$, G. Debras ${ }^{1}$, L. Patissou ${ }^{2}$, R. Parreault ${ }^{2}$, O. Lobios ${ }^{2}$, \\ M. Mangeant ${ }^{2}$, S. Parrot ${ }^{2}$ and O. Hartmann ${ }^{2}$ \\ ${ }^{1}$ CEA, DAM, DI F, 91297 Arpajon, France \\ 2 CEA, DAM, CESTA, 33114 Le Barp, France
}

\begin{abstract}
A Velocity Interferometer for Any Reflector (VISAR) [1, 2] and a Streaked Optical Pyrometer (SOP) [3] were implemented on the "Ligne integration Laser" (LIL) facility. Spatial resolution as good as $10 \mu \mathrm{m}$ in the target plane and velocity resolution as good as $0.1 \mathrm{~km} / \mathrm{s}$ can be achieved. Several campaigns were performed in 2010 involving various experimental setups and physical processes: Boron EOS, Precompress $\mathrm{H} 2$ with special setup of diamond anvil cell and Shock coalescence. This feedback will be of a great help for the Laser Mégajoule facility (LMJ) VISAR design.
\end{abstract}

\section{INTRODUCTION}

High power laser facilities are unequalled experimental tools to study physical properties of matter such as high pressure equation of states or shock timing in the specific framework of inertial confinement fusion experiments. Since 2008, two optical diagnostics were implemented on the "Ligne Integration Laser" (LIL) facility: a Velocity Interferometer for Any Reflector (VISAR) based on two Mach-Zehnder interferometers at two laser wavelengths (1064 and $532 \mathrm{~nm}$ ) and a Streaked Optical Pyrometer (SOP) that measures target self emission in the visible light range and gives access to shock breakout timing and irradiance temperature.

\section{LIL FACILITY SETUP}

The LIL facility is based on four laser beams at $351 \mathrm{~nm}$, delivering a maximum energy of $15 \mathrm{~kJ}$ at the target chamber center. The target chamber is equipped with a full set of visible and X-rays diagnostics. Especially, the VISAR uses a South Pole diagnostic inserter (a). The optical relay can deal with direct (b) and indirect drive (c) experiment setup by rotating its upper part.

\section{DIAGNOSTIC CONCEPT AND PERFORMANCES}

The diagnostic is composed of three main parts (as shown on fig. 2) :

- A probe laser based on a doubled YAG laser with $7 \mathrm{~ns}$ duration pulse. Both fundamental $(1064 \mathrm{~nm})$ and doubled $(532 \mathrm{~nm})$ wavelengths durations are increased to $30 \mathrm{~ns}$ (through an optical fiber setup based on differential lengths between three fibers) and transported with fibers to the optical table in order to be used as light source for the VISARs.

\footnotetext{
ae-mail: stephane.darbon@cea.fr
}

This is an Open Access article distributed under the terms of the Creative Commons Attribution License 2.0, which permits unrestricted use, distribution, and reproduction in any medium, provided the original work is properly cited. 


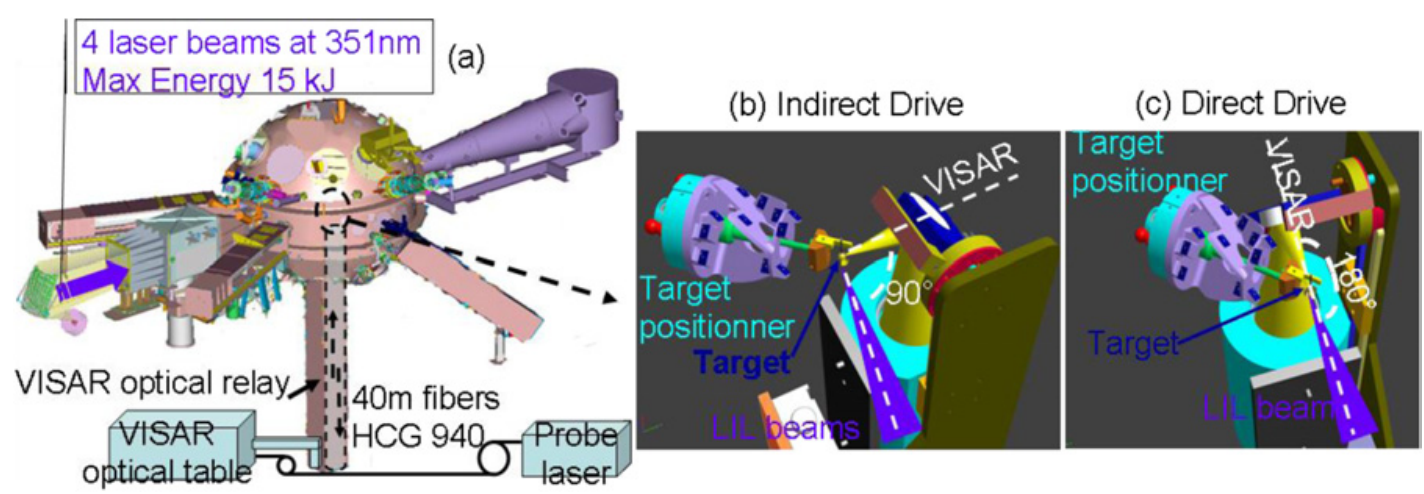

Figure 1. VISAR implementation on LIL Facility target bay (a); Indirect (b) and direct (c) drive configuration.

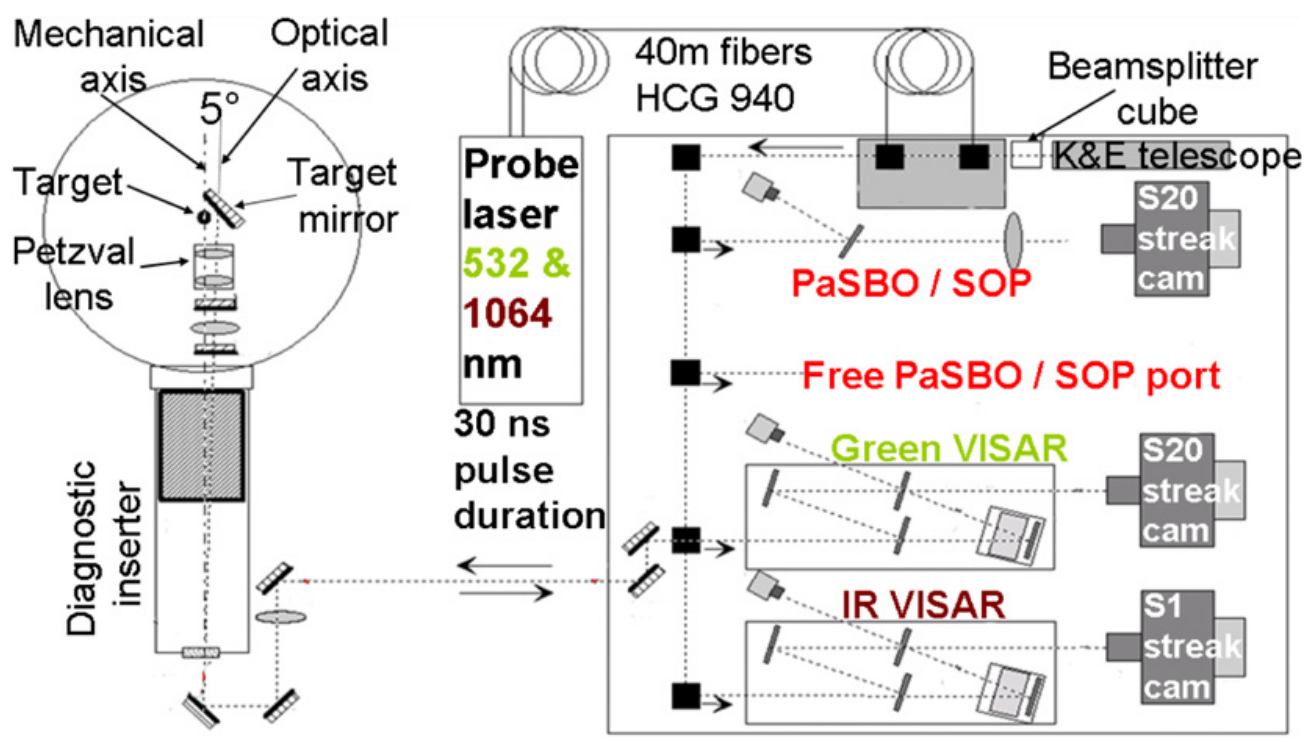

Figure 2. VISAR diagnostic diagram.

- An optical table that hosts an infrared VISAR (equipped with a S1 streak camera), a green VISAR equipped with a S20 streak camera and a Passive Shock Breakout (PaSBO) / SOP working in the 600-700 nm range, equipped with an S20 streak camera.

- An F/3 optical relay of 12 meters length (achieving spatial resolution as good as $10 \mu \mathrm{m}$ in the target plane) that allows the probe laser to illuminate the target and collects the reflected laser light and self emitted light from the target.

Velocity range is between 1 and $100 \mathrm{~km} / \mathrm{s}$ (depending on the etalon size inserted in the interferometer) with a resolution better than $0.1 \mathrm{~km} / \mathrm{s}$, time resolution can vary from 50 to $500 \mathrm{ps}$, depending on the streak sweep time.

\section{FIRST EXPERIMENTS}

\subsection{Shock timing experiment}

In the context of Inertial Confinement Fusion, we aimed at measuring the coalescence time of two shocks with different timings and different intensities [5]. Two shocks were applied to a plastic sample 
(a)

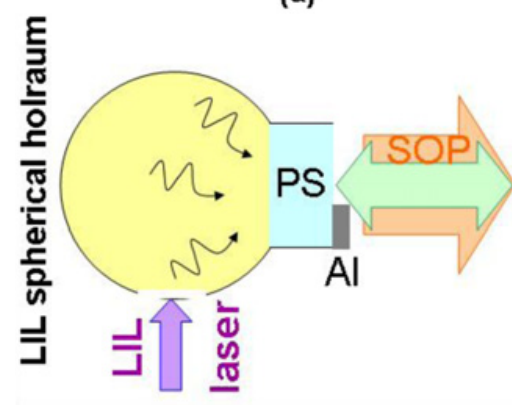

(b)

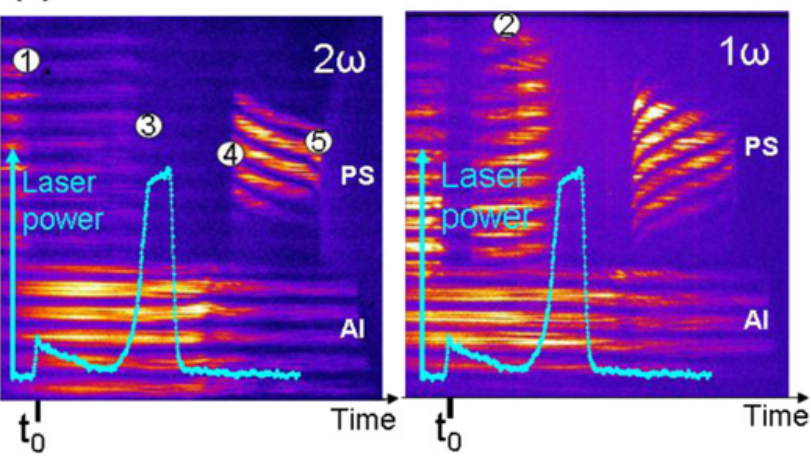

Figure 3. Shock timing experiment configuration (a); $1 \omega$ (b) and $2 \omega$ (c) VISAR signals.

(a)

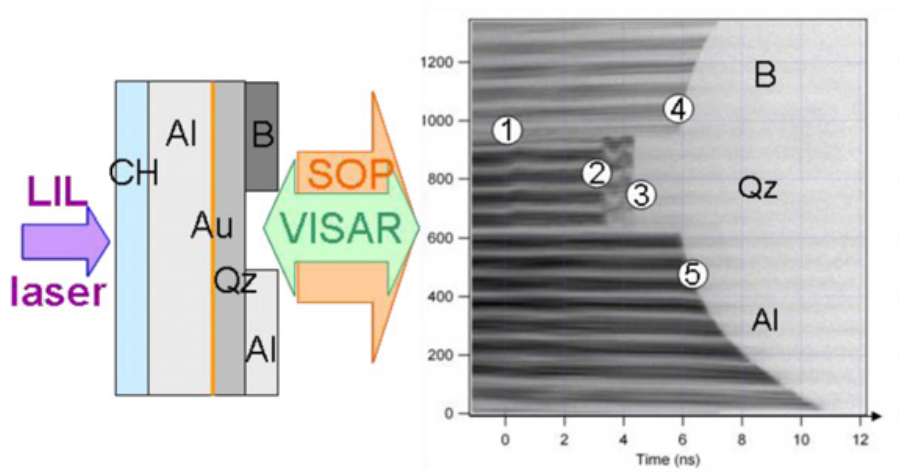

(c)

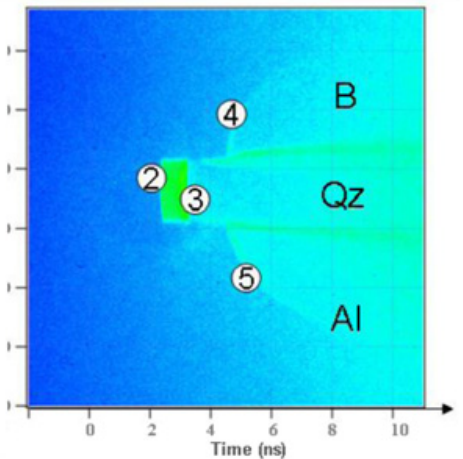

Figure 4. EOS experiment configuration (a); VISAR (b) and SOP (c) signals.

of polystyrene (PS) using indirect drive. Their velocities were recorded with the two VISARs and the SOP, the coalescence time between the two shocks being thus determined. By examining fig. 3(b) and 3(c), we can construct the experiment history. The first shock enters the PS. X-ray preheating induces a loss of reflectivity on both VISARs (1). Then we begin to see the first shock travelling in the PS only with the infrared VISAR (2). The second shock is applied. Again, a loss of reflectivity is due to x-ray preheating (3). Next a jump in reflectivity is observed (4), recovering the signal and allowing to follow the coalesced shock. At the end, we observe the shock breakout (5).

We can see that the reflectivity is not the same at $1 \omega$ and $2 \omega$. The two colours VISARs allow studying the physical properties and behaviour of a material at different wavelengths.

\subsection{EOS experiment}

The goal is to measure the shock velocity and temperature in a reference sample (aluminium) and in a study sample (boron). Then using the Rankine-Hugoniot equations, we can determine the pressure, density and energy in the sample [4].

Using direct drive, a shock is sent into the sample. By examining fig. 4(b) and 4(c), we can construct the experiment history. Small movement of fringes probably due to gold release in quartz (induced by 
X-rays pre-heating) is observed (1). Then the shock enters the reference sample made of Quartz (2). Next we can see the shock breakout of Quartz (3), Boron (4) and Aluminium (5).

From the VISARs, we can follow in-situ the shock velocity with a precision of 1-2\%.

From the SOP signal and the absolute calibration, we can measure either the shock temperature inside the quartz and the time when it exits the aluminium and the boron.

\section{CONCLUSIONS AND PERSPECTIVES}

Each campaign brought a substantial feedback on target design (in order to minimize vulnerability to UV and $\mathrm{x}$ rays) and diagnostic adaptability and operation. This feedback is essential for LMJ VISAR definition.

By the end of 2011, a Gated Optical Imager (GOI) for 2D resolved images of self optical emission at one time (as short as $100 \mathrm{ps)}$ ) and a time fiducial for streak cameras (as precise as $100 \mathrm{ps)} \mathrm{will} \mathrm{be}$ operational.

\section{References}

[1] L.M. Barker, R.E. Hollenbach, J. App Phys. 43(11), 4669 (1972)

[2] P. Celliers et al., Rev. Sci. Instrum. 75, 4916 (2004)

[3] Miller et al., Rev. Sci. Instrum. 78, 034903 (2007)

[4] S. Brygoo et al., APS SCCM09 (2009)

[5] G. Debras et al., these proceedings 